\title{
Antipsychotic-placebo separation on the PANSS-6 subscale as compared to the PANSS-30: a pooled participant-level analysis
}

Fredrik Hieronymus $\mathbb{D I D}^{1,2,3 凶}$, Pernille Kølbæk ${ }^{1,2,4}$, Christoph U. Correll ${ }^{5,6,7}$ and Søren D. Østergaard ${ }^{1,2,4}$

In order for measurement-based care to be implemented, there is a need for brief rating instruments that can be administered in a short amount of time, but that are still sufficiently informative. Here, we assessed the drug-placebo sensitivity of the sixitem subscale (PANSS-6) of the 30-item Positive and Negative Syndrome Scale (PANSS-30) using a large collection of patientlevel data $(n=6685)$ from randomized controlled trials of risperidone and paliperidone. When analyzing the data by study, we found no material difference in mean effect sizes (ES) between the two measures (PANSS-30 ES $=0.45$, PANSS- 6 ES $=0.44$; $p=0.642$ ). Stratifying the pooled population according to several putative effect moderators (e.g., age, formulation, dose, or diagnosis) generally yielded no meaningful ES differences between the two measures. Similarly, early improvement ( $\geq 20 \%$ improvement at week 1 ) on the PANSS- 6 predicted subsequent response ( $\geq 40 \%$ improvement at endpoint) as well as the analog prediction using PANSS-30. Finally, cross-sectional symptom remission assessed via the PANSS- 6 showed very good agreement (sensitivity $=100 \%$, specificity $=98 \%$ ) with cross-sectional symptom remission defined by the Remission in Schizophrenia Working Group.

npj Schizophrenia (2021)7:41 ; https://doi.org/10.1038/s41537-021-00168-x

\section{INTRODUCTION}

The 30 -item Positive and Negative Syndrome Scale (PANSS-30) ${ }^{1}$ is the most widely used rating instrument in schizophrenia. While widespread in research settings, it is not readily amenable to routine clinical use because it takes $45-60 \mathrm{~min}$ to assess all 30 PANSS items ${ }^{1}$. Clinical practice may therefore be better served by using brief rating instruments that can be completed in a short amount of time, e.g., for routine objective tracking of short-term disease progression or improvement, or for assessing sustained response and remission ${ }^{2-4}$.

One such brief rating instrument is the unidimensional six-item PANSS subscale (PANSS-6). Following up on prior item-level analyses of the PANSS ${ }^{5,6}$, the PANSS- 6 was derived as a unidimensional measure of schizophrenia severity via item response theory analyses of the eight-item PANSS-based definition of symptom remission from the Remission in Schizophrenia Working Group ${ }^{2,7}$. The PANSS-6 subscale includes three items measuring positive symptoms (P1 Delusions, P2 Conceptual disorganization, and P3 Hallucinatory behavior) and three items measuring negative symptoms (N1 Blunted affect, N4 Passive/ apathetic social withdrawal, and N6 Lack of spontaneity and flow of conversation). The sensitivity of the PANSS-6, when extracted from PANSS-30 assessments, has previously been found to match that of the PANSS-30 as far as antipsychotic-placebo differences ${ }^{7}$ and differences between antipsychotics are concerned ${ }^{8,9}$. The PANSS- 6 also has a high rate of agreement with the PANSS-based definition of symptom remission from the Remission in Schizophrenia Working Group ${ }^{2,8,9}$. By using the Simplified Negative and Positive Symptoms Interview (SNAPSI) a PANSS-6 rating can be completed in 15-20 $\min ^{10}$. Furthermore, PANSS- 6 ratings obtained using the SNAPSI have been shown to have good inter-rater reliability ${ }^{11,12}$ and validity when using PANSS- 6 ratings obtained via SCI-PANSS of the same patients conducted by independent raters as the reference ${ }^{10}$. Accordingly, PANSS- 6 was recently highlighted as an alternative to longer clinician-rated scales in the practice guideline for the treatment of schizophrenia published by the American Psychiatric Association ${ }^{13}$.

In this study, we compared the sensitivity of PANSS- 6 and PANSS-30 to the efficacy of antipsychotics in a large collection of patient-level data $(n=6685)$ from 18 acute-phase trials of antipsychotics in schizophrenia and schizoaffective disorder, which had used the PANSS-30 as outcome measure. We aimed to assess if there are conditions under which the PANSS- 6 might be less sensitive than the PANSS-30, or conversely, if there are situations in which PANSS- 6 may provide an advantage. Thus, we first compared the PANSS- 6 and PANSS-30 in terms of their sensitivity to drug-placebo differences for all 18 included trials individually. We then pooled all studies and assessed sensitivity across several putative effect moderators (e.g., time under treatment, baseline severity, drug formulation). We also assessed how well cross-sectional symptom remission defined by the PANSS- 6 aligned with the cut-off for symptom remission defined by the Remission in Schizophrenia Working Group ${ }^{2}$, without requiring the 6-month time criterion. Finally, since early symptomatic improvement on the PANSS-30 has been shown to be a strong predictor of subsequent response $\mathrm{e}^{14-17}$, we also assessed the positive predictive value (PPV) and the negative predictive value (NPV) of early improvement on the PANSS- 6 and PANSS-30. We hypothesized that PANSS-6, which can be assessed in much less time than then PANSS-30, would perform on par with PANSS-30, thereby presenting a clinically valid and useful measurement-based care instrument for both clinical care and research purposes.

${ }^{1}$ Department of Clinical Medicine, Aarhus University, Aarhus, Denmark. ${ }^{2}$ Department of Affective Disorders, Aarhus University Hospital-Psychiatry, Aarhus, Denmark. ${ }^{3}$ Institute of Neuroscience and Physiology, University of Gothenburg, Gothenburg, Sweden. ${ }^{4}$ Psychosis Research Unit, Aarhus University Hospital-Psychiatry, Aarhus, Denmark. ${ }^{5}$ Division of Psychiatry Research, The Zucker Hillside Hospital, Glen Oaks, NY, USA. ${ }^{6}$ Department of Psychiatry and Molecular Medicine, Hofstra Northwell School of Medicine, Hempstead, NY, USA. ${ }^{7}$ Department of Child and Adolescent Psychiatry and Psychotherapy, Charité Universitätsmedizin, Berlin, Germany. ${ }^{凶}$ email: fredrik.hieronymus@neuro.gu.se 


\section{RESULTS}

\section{Included studies}

In total, 18 placebo-controlled studies with 46 antipsychotic-placebo comparisons were available for inclusion. Of these, nine investigated paliperidone extended release $(n=3232)$, five investigated paliperidone palmitate $(n=2085)$, three investigated risperidone $(n=$ 1029), and one investigated risperidone depot $(n=336)$. One study, R076477-SCH-302, included elderly patients only; two studies, R076477-PSZ-3001 and RIS-SCH-302, only included adolescents; and the remaining studies included adults. Ten out of 12 studies investigating per oral (PO) formulations were of 6 weeks duration; the remaining 2 were of 4 weeks duration. Four out of six studies investigating long-acting injectables (LAls) were of 13 weeks duration; the remaining two were of 12 and 9 weeks duration, respectively. Details of the included studies are displayed in Table 1.

\section{Study-level comparisons between PANSS-30 and PANSS-6}

Table 2 details all comparisons between active treatment and placebo. Out of 46 antipsychotic-placebo comparisons, a statistically significant superiority of treatment was found for 38 when the PANSS-30 was used as the effect parameter. Likewise, superiority of treatment was found for 39 pairs when the PANSS- 6 was the outcome measure. Seven treatment-placebo comparisons showed no statistically significant separation on either outcome measure and 38 showed statistically significant separation on both outcome measures. The one comparison that differed between outcome measures was the high-dose group (paliperidone extended release 6-12 mg) in Study R076477-PSZ3001, where the PANSS-6 showed a statistically significant superiority of active treatment (ES 0.51, $p=.013$ ), while the PANSS-30 did not (ES 0.33; $p=0.102$ ). Five drug-placebo comparisons showed an ES difference between the PANSS-6 and PANSS-30 above 0.10 (three favoring PANSS-6). Endpoint item scores for these five comparisons are provided in Supplementary Tables 1-5. When analyzing all active treatments in each trial as a group, the arithmetic mean effect size across trials was 0.45 for the PANSS-30 and 0.44 for the PANSS-6; with a non-significant difference in effect size between the two scales of 0.0061 (SEM $0.0130 ; p=0.642$ ). Effect sizes were numerically larger for PANSS30 than for PANSS- 6 in 11 out of 18 trials $(p=0.346)$.

\section{Pooled comparisons between PANSS-30 and PANSS-6}

Table 3 details the results of pooled analyses stratified for putative effect moderators. The overall pooled effect size ( 0.46 for PANSS30 and 0.45 for PANSS-6) was similar to the arithmetic mean effect size. In most analyses (13 out of 18), the PANSS-30 yielded numerically larger effect size. With the exception of the analyses in old age $(\geq 65)$ individuals, where PANSS-6 had an effect size 0.09 units larger than PANSS-30, effect size differences did not exceed 0.03 in favor of either outcome measure.

\section{Comparison of symptomatic remission between PANSS- 6 and PANSS-8}

According to the PANSS-8 symptom remission criteria, $21.6 \%$ of placebo-treated participants and $33.8 \%$ of actively treated participants reached cross-sectional remission (meeting the PANSS-8 symptom remission criteria at the last available visit). Analyses yielded an additional $1.1 \%$ remitted patients on placebo and $1.5 \%$ on active treatment when only the PANSS- 6 criteria were applied. Among these PANSS- 6 remitters, 21 individuals scored 4 points on G5 Mannerisms and posturing, but were otherwise in PANSS-8-defined remission; 67 scored 4 points and 2 scored 5 points on G9 Unusual thought content, but were otherwise in PANSS-8-defined remission, and 4 individuals scored 4 points on both G5 Mannerisms and posturing and G9 Unusual though content. There were thus 94 'false positives' (i.e., remitters on PANSS- 6 but not on PANSS-8), yielding a specificity of $98.0 \%$. Patients in PANSS-6 remission but not in PANSS-8 remission (mean PANSS-30: 69.4) had significantly $(p<0.0001)$ higher PANSS-30 scores than patients in PANSS-8 remission (mean PANSS-30: 56.9 ) but significantly $(p<0.0001)$ lower PANSS-30 scores than patients who were not in remission according to either criteria (mean PANSS-30: 88.2).

\section{Comparisons of PPV and NPV for PANSS-30 and PANSS-6}

Figure $1 \mathrm{a}-\mathrm{h}$ details the PPV and NPV of early response $(\geq 20 \%$ decrease in PANSS-6/PANSS-30 at week 1) as it pertains to ultimate response ( $\geq 40 \%$ decrease in PANSS-6/PANSS-30 at the last available observation). While the NPV was higher for placebotreated patients (82.7-84.8\%; Fig. 1a-d) than for trial participants receiving active treatment (69.3-71.4\%; Fig. 1e-h), the PPV was higher for actively treated participants (54.3-60.6\%; Fig. 1a-d) than for placebo-treated patients (42.0-46.2\%; Fig. 1e-h). There were no major differences in PPV or NPV either across or within PANSS scales, i.e., early improvement on PANSS-6 predicted subsequent response on both the PANSS- 6 and PANSS-30 with comparable accuracy, and early improvement on the PANSS-30 likewise predicted subsequent response on both the PANSS- 6 and PANSS-30 with comparable accuracy.

\section{Effect sizes for individual PANSS items}

Table 4 contains the results from the post hoc analysis of effect sizes for individual PANSS items. Most effect sizes (26/30) were in the range of $0.20-0.40$. The lowest effect size was observed for item G7 Motor retardation (ES 0.11), and the highest effect sizes were seen for the two positive symptoms P2 Conceptual disorganization and P6 Suspiciousness/persecution (ES 0.40 for both items). The impact of adding a specific item to the PANSS- 6 largely mirrored effect sizes for the individual items. The largest improvement in subscale effect size $(4.6 \%)$ was seen for item P7 Hostility, and the largest decline $(-4.1 \%)$ was seen for item G7 Motor retardation.

\section{DISCUSSION}

The main finding of this study is that the PANSS- 6 and the PANSS30 have comparable sensitivity to antipsychotic efficacy across a range of putative effect moderators. This finding was evidenced by a negligible difference in mean effect sizes when all 18 included trials were analyzed individually; likewise, subgroup analyses showed no effect size differences exceeding 0.03 , with the exception of the old age $(\geq 65)$ subgroup where PANSS-6 showed an ES 0.09 units larger than PANSS-30. Similarly, agreement between cross-sectional symptom remission as defined by PANSS- 6 and by the eight-item definition suggested by the Remission in Schizophrenia Working Group ${ }^{2}$ was very high. Moreover, with regard to prediction of subsequent response via early improvement, the PPV and NPV were comparable between the PANSS- 6 and the PANSS-30, both within and across outcome scales. That PANSS-6 is equally sensitive to the PANSS-30 with regard to the efficacy of antipsychotics is in line with results from prior studies on both the efficacy ${ }^{7}$ and effectiveness of antipsychotics in the treatment of schizophrenia ${ }^{9,10}$.

While, based on these and previous results, PANSS- 6 seems to be an adequately sensitive instrument for tracking core schizophrenia severity, it should be emphasized that PANSS-6 ratings might need to be accompanied by ratings on measures of other constructs that are relevant in relation to the care of individuals with schizophrenia, e.g., depression, anxiety, cognition, agitation/ aggression, medication side effects, level of functioning and 


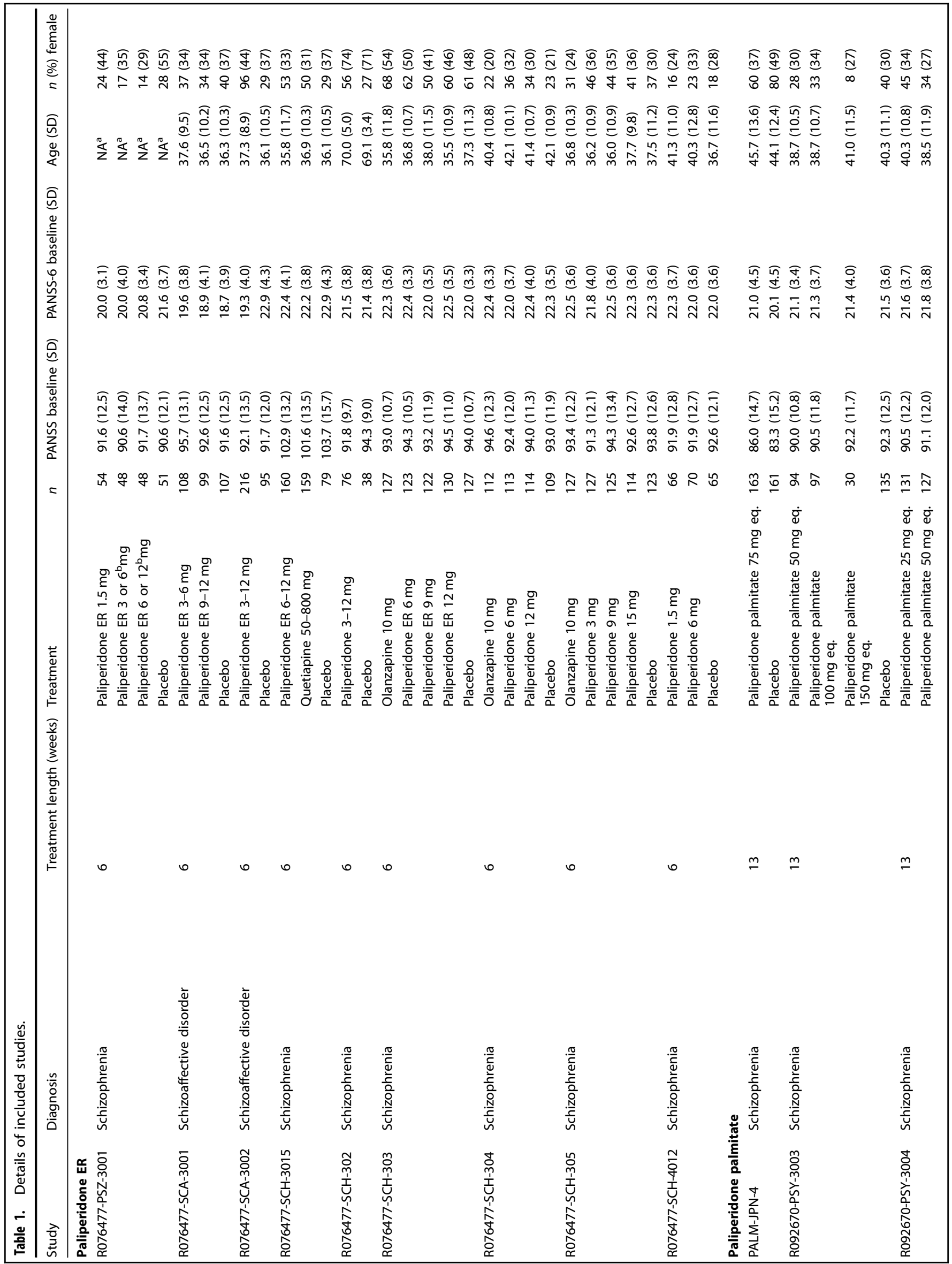




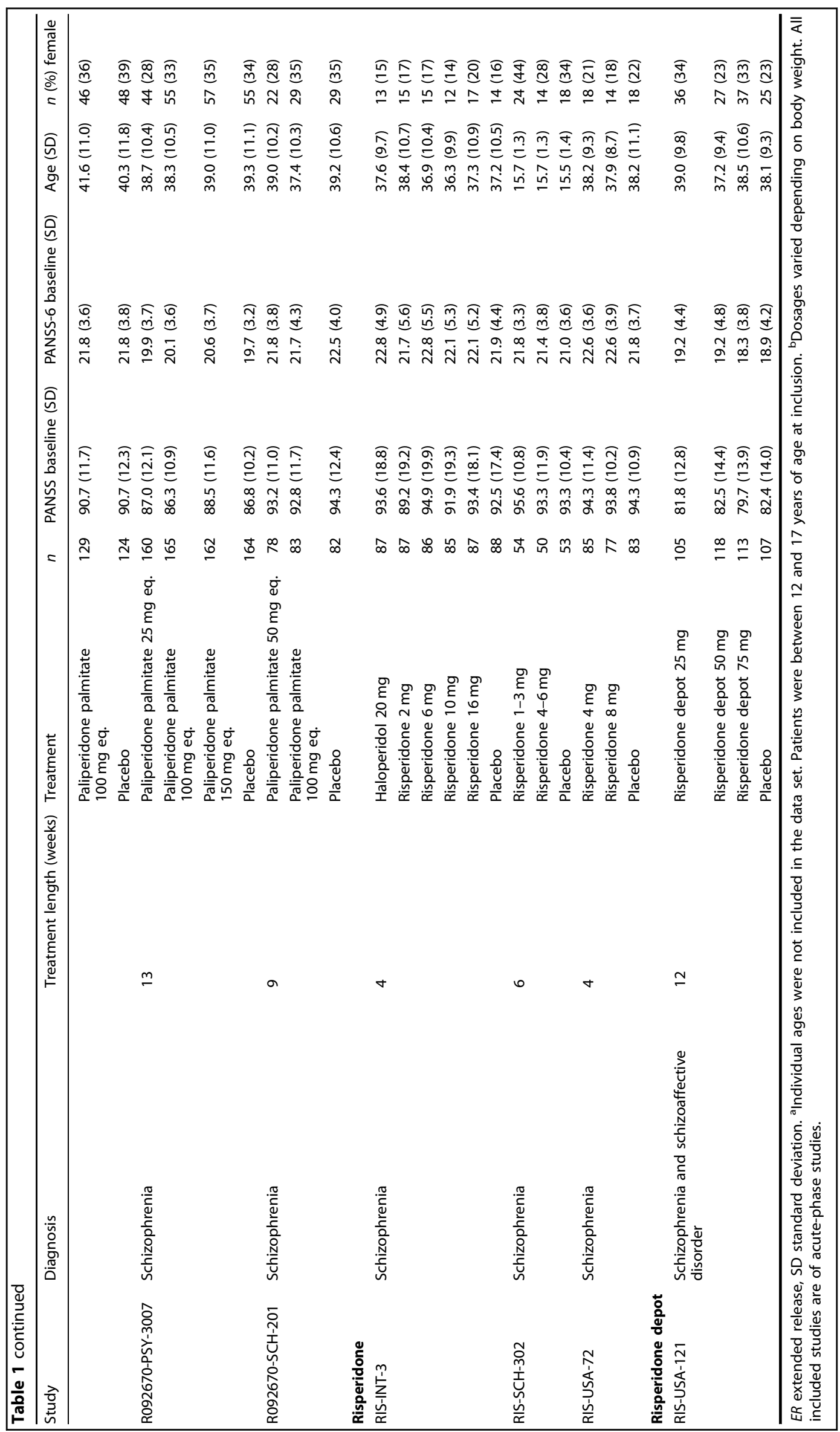




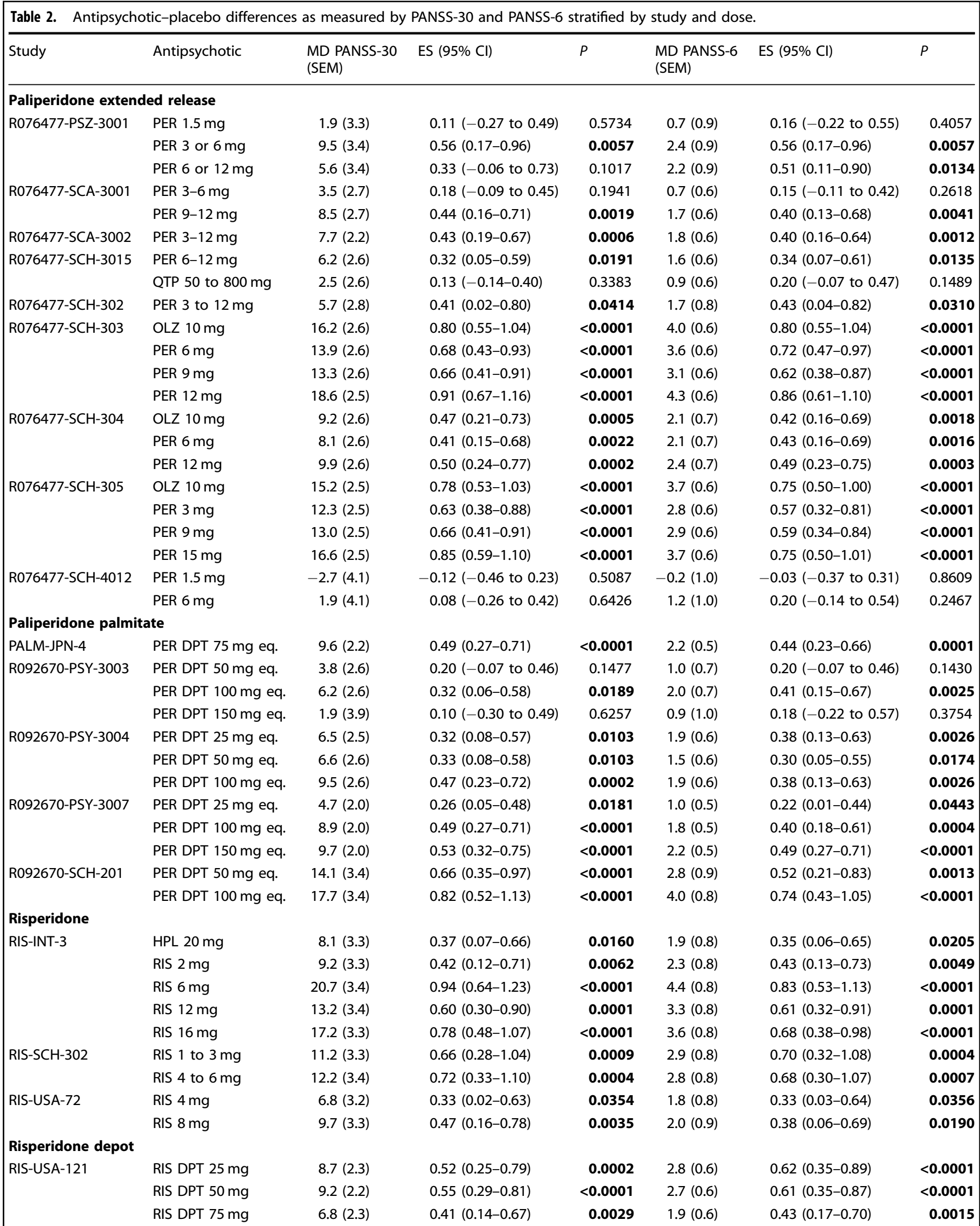

Significant contrasts are denoted in bold. DPT depot formulation, ES effect size, HPL haloperidol, MD mean difference, OLZ olanzapine, PER paliperidone extended release, QTP quetiapine, RIS risperidone, SEM standard error of the mean. 


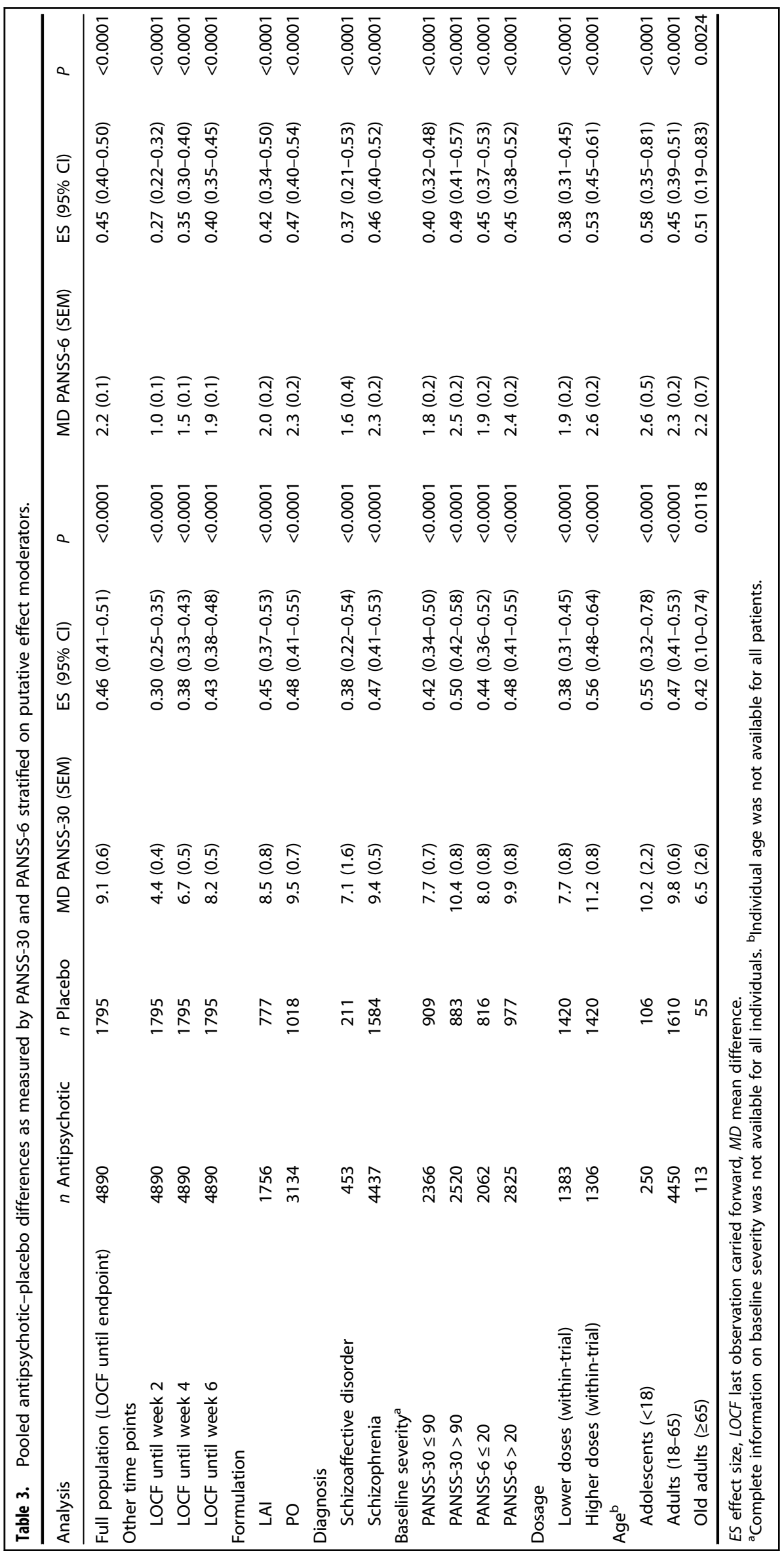



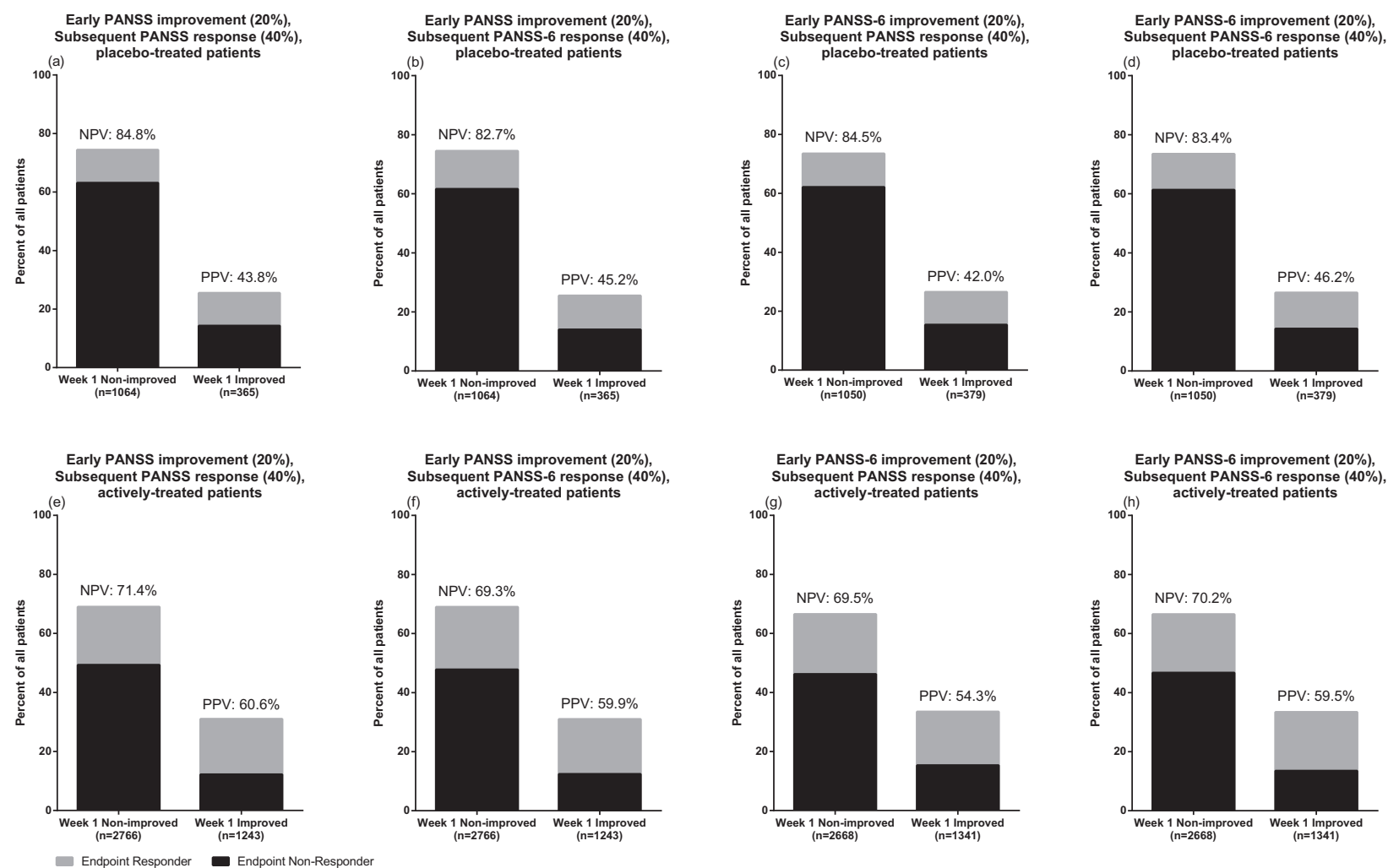

Fig. 1 Positive and negative predictive value of early improvement on subsequent response. NPV negative predictive value, PPV positive predictive value.

quality of life $\mathrm{e}^{18-22}$, and that such considerations will depend on the research questions and areas of individual need being addressed.

With regard to prediction of subsequent response via early improvement, the PPV and NPV of the PANSS-6 were comparable to those of the PANSS-30, both with respect to longitudinal prediction based on the same measure and in comparisons across time and between the PANSS-6 and PANSS-30 (Fig. 1). These results replicate previous findings showing that early improvement on the PANSS-30, as well as on other schizophrenia rating scales ${ }^{14-17}$, is a strong predictor of subsequent response. The fact that this relationship holds also for PANSS- 6 is of obvious importance if the PANSS- 6 - which does not take as much time to complete as the PANSS-30 - is to be used to reduce contact time in clinical trials that may contribute to an observed inflated placebo response ${ }^{23}$, or to inform personalized treatment in clinical care settings.

The high rate of agreement (100\% sensitivity, $98.0 \%$ specificity) between cross-sectional symptom remission as defined by the PANSS-8 and PANSS- 6 is partly by design since all patients meeting the PANSS-8 criteria also qualify for remission according to the PANSS-6, thus yielding perfect sensitivity. Patients who had remitted according to PANSS-6, but not PANSS-8, had significantly higher PANSS-30 symptom scores than PANSS-8 remitters (69.4 vs 56.9) but significantly lower symptom scores than non-remitters to the PANSS-8 definition (69.4 vs 88.2). This finding suggests that the small fraction of additional PANSS- 6 remitters may differ from the larger group of PANSS-8 remitters.

Most individual PANSS items ( 26 out of 30 ) had effect sizes in the range of $0.20-0.40$. As expected due to the predominant efficacy of currently available antipsychotics for positive rather than negative symptoms ${ }^{24,25}$, positive symptoms were, on average, those that separated most clearly from placebo (Table 4). This contrasts to similar analyses of patients with major depression where much larger disparities in individual-item effect sizes have been reported for the Hamilton Depression Rating Scale ${ }^{26}$. Notably, the two PANSS items with the lowest effect sizes were G7 Motor retardation (ES 0.11 ) and G1 Somatic concern (ES 0.15), which could reflect that specific side effects of antipsychotics worked against a general improvement in the underlying condition ${ }^{27,28}$, as has been suggested for depression-i.e., that some side effects (e.g., sedation, dystonia, arthralgia, nausea) are mistaken for psychopathology as measured by G7 Motor retardation and G1 Somatic concern ${ }^{29-31}$. Another factor that may contribute to the low drug-placebo separation for these items is the comparatively low baseline scores ( 2.30 for G7 and 2.54 for G1) in combination with the fact that these symptoms showed some improvement also on placebo (endpoint scores on placebo: 2.10 and 2.35, respectively). Taken together, this leaves little room for a true drug effect to be detected.

This study has a number of limitations. First, although we included a large number of trials and participants, only few trials included certain subgroups, e.g., trials of schizoaffective disorder and trials focusing on adolescents or older adults. The power to detect differences in sensitivity between the PANSS- 6 and the PANSS-30 in these subgroups was hence insufficient, and the results should be interpreted with caution. Second, the results stem from data obtained through randomized clinical trials, and it remains to be investigated to what degree these results will generalize to clinical settings. Third, PANSS- 6 ratings were derived from the full PANSS-30 ratings; however, PANSS-6 ratings obtained using the SNAPSI may not correspond to those observed when conducting the full SCI-PANSS. Ideally, one should compare data from different raters independently scoring the PANSS- 6 and PANSS-30 in the same patients, at the same time. In lieu of such data, analyses extracting PANSS-6 scores from full PANSS-30 
F. Hieronymus et al.

Table 4. Antipsychotic-placebo differences on individual PANSS-30 items (pooled population).

\begin{tabular}{|c|c|c|c|c|c|c|c|c|}
\hline \multirow[b]{2}{*}{ Item } & \multicolumn{2}{|c|}{ Baseline score } & \multicolumn{2}{|c|}{ Endpoint score } & \multirow[b]{2}{*}{ ES $(95 \% \mathrm{Cl})$} & \multirow[b]{2}{*}{$P$} & \multicolumn{2}{|c|}{$\begin{array}{l}\text { If included in } \\
\text { PANSS-6 }\end{array}$} \\
\hline & Placebo & Antipsychotic & Placebo & Antipsychotic & & & ES & $\%$ ES-diff \\
\hline P2 Conceptual disorganization & 3.5 & 3.6 & 3.3 & 2.9 & $0.40(0.35-0.45)$ & $<0.0001$ & NA & NA \\
\hline P3 Hallucinatory behavior & 3.7 & 3.7 & 3.2 & 2.7 & $0.36(0.31-0.41)$ & $<0.0001$ & NA & NA \\
\hline P6 Suspiciousness/persecution & 3.9 & 3.9 & 3.4 & 2.9 & $0.40(0.35-0.45)$ & $<0.0001$ & 0.46 & $3.1 \%$ \\
\hline P7 Hostility & 2.5 & 2.5 & 2.4 & 2.0 & $0.38(0.33-0.43)$ & $<0.0001$ & 0.47 & $4.6 \%$ \\
\hline N1 Blunted affect & 3.3 & 3.4 & 3.1 & 2.9 & $0.21(0.16-0.26)$ & $<0.0001$ & NA & NA \\
\hline N2 Emotional withdrawal & 3.5 & 3.6 & 3.3 & 3.0 & $0.28(0.23-0.33)$ & $<0.0001$ & 0.44 & $-1.9 \%$ \\
\hline N3 Poor rapport & 2.9 & 3.0 & 2.8 & 2.5 & $0.35(0.30-0.40)$ & $<0.0001$ & 0.46 & $1.5 \%$ \\
\hline G1 Somatic concern & 2.5 & 2.6 & 2.4 & 2.2 & $0.15(0.10-0.20)$ & $<0.0001$ & 0.44 & $-2.4 \%$ \\
\hline G2 Anxiety & 3.2 & 3.2 & 2.9 & 2.6 & $0.28(0.23-0.33)$ & $<0.0001$ & 0.45 & $0.5 \%$ \\
\hline G3 Guilt feelings & 2.3 & 2.2 & 1.9 & 1.8 & $0.18(0.13-0.23)$ & $<0.0001$ & 0.45 & $-0.1 \%$ \\
\hline G4 Tension & 3.1 & 3.1 & 2.8 & 2.4 & $0.32(0.27-0.37)$ & $<0.0001$ & 0.46 & $1.8 \%$ \\
\hline G5 Mannerisms and posturing & 2.4 & 2.4 & 2.3 & 2.09 & $0.22(0.17-0.27)$ & $<0.0001$ & 0.45 & $-0.7 \%$ \\
\hline G6 Depression & 2.7 & 2.6 & 2.4 & 2.2 & $0.18(0.13-0.23)$ & $<0.0001$ & 0.44 & $-1.6 \%$ \\
\hline G7 Motor retardation & 2.3 & 2.3 & 2.1 & 2.0 & $0.11(0.06-0.16)$ & $<0.0001$ & 0.43 & $-4.1 \%$ \\
\hline G8 Uncooperativeness & 2.3 & 2.3 & 2.4 & 2.0 & $0.32(0.27-0.37)$ & $<0.0001$ & 0.46 & $1.7 \%$ \\
\hline G9 Unusual thought content & 3.5 & 3.6 & 3.2 & 2.9 & $0.30(0.25-0.35)$ & $<0.0001$ & 0.45 & $-0.2 \%$ \\
\hline G10 Disorientation & 2.1 & 2.1 & 2.0 & 1.8 & $0.23(0.18-0.28)$ & $<0.0001$ & 0.45 & $1.1 \%$ \\
\hline
\end{tabular}

ratings are the best alternative. Notably, we recently conducted a study comparing dedicated PANSS- 6 assessments via the SNAPSI and PANSS- 6 assessments as part of the full PANSS-30 ratings obtained by independent raters and found good agreement across the two methods of obtaining PANSS- 6 ratings ${ }^{11}$. Finally, from an implementation perspective, it should be noted that, while the SNAPSI is freely available for non-commercial clinical and research purposes (https://www.medavante-prophase.com/ welcome-to-snapsi/), use of the PANSS-30 and its subscales (including the PANSS-6) requires a license agreement with the copyright holder (Multi-Health Systems) and is associated with a fee.

To summarize, in this large-scale patient-level analysis, the sensitivity of the PANSS- 6 to antipsychotic efficacy was comparable to that of the PANSS-30 across a range of different tests and putative effect moderators. These findings add to a growing literature indicating that the PANSS- 6 can be used to adequately monitor the severity of core schizophrenia symptomatology over time ${ }^{7-13}$. Given its brevity, the PANSS-6 may facilitate the implementation of objective tracking of core schizophrenia severity in the clinic and contribute to making future clinical trials of treatments for schizophrenia less costly and resource intensive.

\section{METHODS}

\section{Data acquisition}

We requested patient-level data for all industry-sponsored, acute-phase, placebo-controlled trials of risperidone and paliperidone via the Yale Open Data Access (YODA) project ${ }^{32}$. Remote access to patient-level data was provided by Johnson \& Johnson and YODA for all 19 requested studies. One study (RIS-USA-1/Study 201) used the Brief Psychiatric Rating Scale (BPRS) instead of the PANSS and could hence not be included in the analyses. In order to verify the accuracy of the data, we compared our results to those from study reports provided by YODA and with those available in public reports from the United States Food and Drug Administration ${ }^{33-39}$, the European Medicines Agency ${ }^{40}$, and ClinicalTrials.gov ${ }^{41-43}$

\section{Analyses and statistics}

Individual antipsychotics and doses were first analyzed by trial using analysis of covariance (ANCOVA). Analyses were performed on the intention-to-treat population using last observation carried forward methodology up until the last scheduled evaluation for each trial. The efficacy of all included treatment arms was assessed using both the PANSS-30 and PANSS-6. The models included a fixed factor for treatment and baseline score on the corresponding outcome measure as a covariate. 
Effect size differences between the PANSS- 6 and PANSS-30 were assessed using paired samples $t$-test, and the rate at which effect sizes favored either outcome measure over the other was assessed using the onesample chi-squared test with both outcomes expected to occur with equal frequency. In order to not include placebo-treated participants more than once (i.e., since a trial may have had several active treatment arms), the two latter analyses were conducted with all patients receiving active treatment analyzed together in each trial.

We then pooled all available studies and conducted analyses stratified by putative effect moderators. The model specifications for the pooled analyses were analogous to those used for the analyses of individual studies with the addition of a fixed factor for study (with one exception, see below). The assessed effect moderators were earlier endpoints (weeks 2,4 , and 6), drug formulation ( $\mathrm{LAl}$, or PO), diagnosis (schizophrenia vs. schizoaffective disorder), baseline severity (PANSS-30/PANSS-6 at or below median vs. above median), dose, and age group (adolescents, adults, older adults). For the dose analyses, we included all trials investigating at least two different doses of one active treatment and included the arm with the lowest given dose in the 'low-dose' group and the arm with the highest given dose in the 'high-dose' group. For the age group analyses, we excluded the study factor since some studies included very few patients belonging to a specific age group.

We then assessed endpoint symptom remission in the pooled population (i.e., a cross-sectional definition of remission not requiring the 6-month time criterion) $)^{2}$. We did so by contrasting PANSS-6-defined remission (defined as a score of $\leq 3$ on all PANSS- 6 items, range 1-7) with the eight-item definition (defined as a score of $\leq 3$ on all PANSS- 6 items as well as on G5 Mannerisms and posturing and G9 Unusual thought content, PANSS-8) suggested by the Remission in Schizophrenia Working Group ${ }^{2}$. Due to the overlap between the criteria, all patients in remission according to the PANSS-8 criteria were by definition also in PANSS-6-defined remission. We thus focused on those additional patients found to be in remission only according to the PANSS-6. We detailed their scores on the two additional items in the PANSS- 8 and assessed (via independent samples $t$-tests) whether their PANSS-30 scores were different from those of patients who were in symptom remission according to the PANSS-8 definition, and from those of patients who were not in remission according to either definition, respectively ${ }^{2}$.

We then assessed the PPV and NPV of early improvement (defined as a $\geq 20 \%$ reduction in PANSS- 6 or PANSS-30 at the week 1 evaluation) on subsequent response (defined as a $\geq 40 \%$ reduction in PANSS- 6 or PANSS30 at the endpoint evaluation). These assessments were performed both within scales (e.g., early improvement in PANSS-6 predicting PANSS-6 response) and across scales (e.g., early improvement in PANSS-30 predicting PANSS-6 response). Analyses were stratified by treatment (placebo or active treatment). For patients to be included in the analyses, they needed to have an evaluation during week 1 and at least one subsequent evaluation. The last available scheduled evaluation was used as the endpoint evaluation. In order for percentage differences to make intuitive sense, PANSS scores were rescaled from 1 to 7 to 0 to 6 (i.e., so that a patient with no PANSS-30-measured symptoms would score 0 rather than 30) for this analysis.

Finally, based on the observation that the pooled effect sizes obtained with the PANSS- 6 and PANSS-30 were almost identical, but slightly higher for the PANSS-30, we conducted the following post hoc analyses. First, we calculated effect sizes for all individual PANSS-30 items. Subsequently, we analyzed how the drug-placebo sensitivity of the PANSS- 6 would be impacted by including each of the 24 PANSS-30 items not included in the PANSS-6 ("add-one-in" analysis). The models used for these analyses were identical to those described above but with the outcome parameter being the item in question or that item plus PANSS-6, with the baseline score on the respective outcome parameter being included as a covariate.

All analyses were conducted using $\mathrm{R}$ version 3.6.1. Two-sided $p$ values $<0.05$ were considered statistically significant. Due to substantial overlap between outcomes (the PANSS- 6 items are nested within the PANSS-30), populations (individual trials are nested within the pooled population), and subgroups (e.g., participants with low scores on the PANSS- 6 also tend to have low scores on the PANSS-30), correction for multiple testing was not performed.

\section{Ethics}

The data used for this study consist of de-identified patient-level data from previously conducted clinical trials. Secondary analyses of de-identified data does not fall under the purview of ethical committees in the jurisdiction where the research was carried out.

\section{Reporting summary}

Further information on research design is available in the Nature Research Reporting Summary linked to this article.

\section{DATA AVAILABILITY}

The data used in this article can be requested from the Yale Open Data Access website ${ }^{32}$.

\section{CODE AVAILABILITY}

No non-standard code or algorithms were used in the generation of the results in this article.

Received: 2 March 2021; Accepted: 20 July 2021; Published online: 27 August 2021

\section{REFERENCES}

1. Kay, S. R., Fiszbein, A. \& Opler, L. A. The Positive And Negative Syndrome Scale (PANSS) for schizophrenia. Schizophr. Bull. 13, 261-276 (1987).

2. Andreasen, N. C. et al. Remission in schizophrenia: proposed criteria and rationale for consensus. Am. J. Psychiatry 162, 441-449 (2005).

3. Correll, C. U., Kishimoto, T., Nielsen, J. \& Kane, J. M. Quantifying clinical relevance in the treatment of schizophrenia. Clin. Ther. 33, B16-B39 (2011).

4. Lindenmayer, J. P. Are shorter versions of the Positive And Negative Syndrome Scale (PANSS) Doable? A critical review. Innov. Clin. Neurosci. 14, 73-76 (2017).

5. Levine, S. Z. \& Rabinowitz, J. An item response theory analysis of the Positive And Negative Syndrome Scale: psychometric recommendations to improve the appraisal of symptom severity. Schizophr. Res. 102, 227-227 (2008).

6. Levine, S. Z., Rabinowitz, J. \& Rizopoulos, D. Recommendations to improve the Positive And Negative Syndrome Scale (PANSS) based on item response theory. Psychiatry Res. 188, 446-452 (2011).

7. Østergaard, S. D., Lemming, O. M., Mors, O., Correll, C. U. \& Bech, P. PANSS-6: a brief rating scale for the measurement of severity in schizophrenia. Acta Psychiatr. Scand. 133, 436-444 (2016).

8. Ostergaard, S. D., Foldager, L., Mors, O., Bech, P. \& Correll, C. U. The validity and sensitivity of PANSS- 6 in the Clinical Antipsychotic Trials of Intervention Effectiveness (CATIE) Study. Schizophr. Bull. 44, 453-462 (2018).

9. Ostergaard, S. D., Foldager, L., Mors, O., Bech, P. \& Correll, C. U. The validity and sensitivity of PANSS-6 in treatment-resistant schizophrenia. Acta Psychiatr. Scand. 138, 420-431 (2018).

10. Kølbæk, P. et al. Clinical validation of ratings on the six-item Positive And Negative Syndrome Scale (PANSS-6) obtained via the Simplified Negative And Positive Symptoms Interview (SNAPSI). J. Psychopharmacol. (2021) (Advance online publication). https://doi.org/10.1177/0269881121996890.

11. Kolbaek, P. et al. Inter-rater reliability of ratings on the six-item Positive And Negative Syndrome Scale (PANSS-6) obtained using the Simplified Negative and Positive Symptoms Interview (SNAPSI). Nord J. Psychiatry 72, 431-436 (2018).

12. Kølbæk, P. et al. Standardized training in the rating of the six-item Positive And Negative Syndrome Scale (PANSS-6). Schizophr. Res 228, 438-446 (2021).

13. American Psychiatric Association. The American Psychiatric Association Practice Guideline for the Treatment of Patients with Schizophrenia 3rd edn (American Psychiatric Publishing, 2021).

14. Kinon, B. J. et al. Early response to antipsychotic drug therapy as a clinical marker of subsequent response in the treatment of schizophrenia. Neuropsychopharmacology 35, 581-590 (2010).

15. Correll, C. U., Malhotra, A. K., Kaushik, S., McMeniman, M. \& Kane, J. M. Early prediction of antipsychotic response in schizophrenia. Am. J. Psychiatry 160, 2063-2065 (2003).

16. Lin, C. H. et al. Early improvement in PANSS-30, PANSS-8, and PANSS-6 scores predicts ultimate response and remission during acute treatment of schizophrenia. Acta Psychiatr. Scand. 137, 98-108 (2018).

17. Samara, M. T. et al. Early improvement as a predictor of later response to antipsychotics in schizophrenia: a diagnostic test review. Am. J. Psychiatry 172, 617-629 (2015).

18. Addington, D., Addington, J. \& Atkinson, M. A psychometric comparison of the Calgary Depression Scale for Schizophrenia and the Hamilton Depression Rating Scale. Schizophr. Res. 19, 205-212 (1996). 
19. Llorca, P.-M. et al. A composite scale applied to evaluate anxiety in schizophrenic patients (SAES). Eur. Arch. Psychiatry Clin. Neurosci. 264, 171-178 (2014).

20. Lingjaerde, O., Ahlfors, U. G., Bech, P., Dencker, S. J. \& Elgen, K. The UKU side effect rating scale. A new comprehensive rating scale for psychotropic drugs and a cross-sectional study of side effects in neuroleptic-treated patients. Acta Psychiatr. Scand. 334, 1-100 (1987).

21. Waddell, L. \& Taylor, M. A new self-rating scale for detecting atypical or secondgeneration antipsychotic side effects. J. Psychopharmacol. 22, 238-243 (2008).

22. Topp, C. W., Østergaard, S. D., Søndergaard, S. \& Bech, P. The WHO-5 Well-Being Index: a systematic review of the literature. Psychother. Psychosomatics 84, 167-176 (2015).

23. Gopalakrishnan, M. et al. The trend of increasing placebo response and decreasing treatment effect in schizophrenia trials continues: an update from the US Food and Drug Administration. J. Clin. Psychiatry 81, 19r12960 (2020).

24. Huhn, M. et al. Comparative efficacy and tolerability of 32 oral antipsychotics for the acute treatment of adults with multi-episode schizophrenia: a systematic review and network meta-analysis. Lancet 394, 939-951 (2019).

25. Krause, M. et al. Antipsychotic drugs for patients with schizophrenia and predominant or prominent negative symptoms: a systematic review and meta-analysis. Eur. Arch. Psychiatry Clin. Neurosci. 268, 625-639 (2018).

26. Hieronymus, F., Emilsson, J. F., Nilsson, S. \& Eriksson, E. Consistent superiority of selective serotonin reuptake inhibitors over placebo in reducing depressed mood in patients with major depression. Mol. Psychiatry 21, 523-530

(2016).

27. Mishara, A. L. \& Goldberg, T. E. A meta-analysis and critical review of the effects of conventional neuroleptic treatment on cognition in schizophrenia: opening a closed book. Biol. Psychiatry 55, 1013-1022 (2004).

28. Correll, C. U. \& Schooler, N. R. Negative symptoms in schizophrenia: a review and clinical guide for recognition, assessment, and treatment. Neuropsychiatr. Dis. Treat. 16, 519-534 (2020).

29. Bech, P. Is the antidepressive effect of second-generation antidepressants a myth? Psychol. Med 40, 181-186 (2010).

30. Ostergaard, S. D. Do not blame the SSRIs: blame the Hamilton Depression Rating Scale. Acta Neuropsychiatr. 30, 241-243 (2018).

31. Ostergaard, S. D. Optimal dosing of antidepressant drugs. Lancet Psychiatry 6, 804-805 (2019)

32. Yale Open Data Access Project. https://yoda.yale.edu/ (2020).

33. United States Food \& Drug Administration. Drug Approval Package-Risperdal. http://breggin.com/td-resources/Risperdal-new-drug-application.pdf (1993).

34. United States Food \& Drug Administration. Drug Approval Package-Risperdal, Supplemental Application for Schizophrenia in Adolescents. https://www. accessdata.fda.gov/drugsatfda_docs/nda/2007/020272Orig1s046-part1.pdf (2007).

35. United States Food \& Drug Administration. Drug Approval Package-Risperdal, Supplemental Application for Once-Daily Dosing. https://www.accessdata.fda. gov/drugsatfda_docs/nda/97/20588.pdf (1997).

36. United States Food \& Drug Administration. Drug Approval Package-Risperdal Consta. https://www.accessdata.fda.gov/drugsatfda_docs/nda/2003/21346_Risperdal \%20Consta\%20Long-Acting\%20Injection_medr.PDF (2003).

37. United States Food \& Drug Administration. Drug Approval Package-Invega. https://www.accessdata.fda.gov/drugsatfda_docs/nda/2006/021999s000_TOC.cfm (2006).

38. United States Food \& Drug Administration. Clinical Review-Invega, Adolescents. https://www.fda.gov/media/80425/download (2011).

39. United States Food \& Drug Administration. Medical Review-Invega Sustenna. https://www.accessdata.fda.gov/drugsatfda_docs/nda/2009/022264s000medr.pdf (2009).

40. European Medicines Agency. Assessment Report-Invega, Schizoaffective Disorder. https://www.ema.europa.eu/en/documents/variation-report/invega-h-c746-ii-0023-epar-assessment-report-variation_en.pdf (2010).

41. Janssen Pharmaceutical K.K. Clinical Study Report-PALM-JPN-4 (NCT01299389). https://clinicaltrials.gov/ct2/show/results/NCT01299389 (2013).

42. Johnson \& Johnson Pharmaceutical Research \& Development LLC. Clinical Study Report-R076477-SCH-4012 (NCT00524043). https://clinicaltrials.gov/ct2/show/ results/NCT00524043 (2014).

43. Johnson \& Johnson Pharmaceutical Research \& Development LLC. Clinical Study Report-R076477-SCH-3015 (NCT00334126). https://clinicaltrials.gov/ct2/show/ record/NCT00334126 (2014).

\section{ACKNOWLEDGEMENTS}

This study, carried out under YODA Project 2019-3941, used data obtained from the Yale University Open Data Access Project, which has an agreement with JANSSEN RESEARCH \& DEVELOPMENT, L.L.C. The interpretation and reporting of research using this data are solely the responsibility of the authors and does not necessarily represent the official views of the Yale University Open Data Access Project or JANSSEN RESEARCH \& DEVELOPMENT, L.L.C. This work was supported by a grant from the Lundbeck Foundation (grant number R303-2018-3429) to F.H. SD $\varnothing$ is supported by grants from the Novo Nordisk Foundation (grant number NNF20SA0062874); the Lundbeck Foundation (grant numbers R358-2020-2341, R344-2020-1073); the Danish Cancer Society (grant number R283-A16461); the Central Denmark Region Fund for Strengthening of Health Science (grant number: 1-36-72-4-20), the Danish Agency for Digitisation Investment Fund for New Technologies (grant number 2020-6720) and Independent Research Fund Denmark (grant number 7016-00048B).

\section{AUTHOR CONTRIBUTIONS}

All authors made substantial contributions to the conception and design of the work. F.H. drafted the manuscript and conducted the analyses. All authors contributed to the interpretation of data, critically reviewed and revised the manuscript, and have given their approval for the final version to be submitted for publication.

\section{FUNDING}

Open access funding provided by University of Gothenburg.

\section{COMPETING INTERESTS}

C.U.C. has been a consultant and/or advisor to or has received honoraria from Acadia, Alkermes, Allergan, Angelini, Axsome, Gedeon Richter, Gerson Lehrman Group, IntraCellular Therapies, Janssen/J\&J, LB Pharma, Lundbeck, MedAvante-ProPhase, Medscape, Mylan, Neurocrine, Noven, Otsuka, Pfizer, Recordati, Rovi, Sumitomo Dainippon, Sunovion, Supernus, Takeda, and Teva. He has provided expert testimony for Janssen and Otsuka. He served on a Data Safety Monitoring Board for Lundbeck, Rovi, Supernus, and Teva. He received royalties from UpToDate and grant support from Janssen and Takeda. He is also a stock option holder of LB Pharma. S.D.Ø. received the 2020 Lundbeck Foundation Young Investigator Prize. The remaining authors declare no competing interests. Aarhus University, The Feinstein Institute for Medical Research, and MedAvante-ProPhase Inc. each hold one-third of the copyright for the Simplified Negative and Positive Symptoms Interview (SNAPSI).

\section{ADDITIONAL INFORMATION}

Supplementary information The online version contains supplementary material available at https://doi.org/10.1038/s41537-021-00168-x.

Correspondence and requests for materials should be addressed to F.H.

Reprints and permission information is available at http://www.nature.com/ reprints

Publisher's note Springer Nature remains neutral with regard to jurisdictional claims in published maps and institutional affiliations.

\begin{abstract}
Attribution 4.0 International License, which permits use, sharing, adaptation, distribution and reproduction in any medium or format, as long as you give appropriate credit to the original author(s) and the source, provide a link to the Creative Commons license, and indicate if changes were made. The images or other third party material in this article are included in the article's Creative Commons license, unless indicated otherwise in a credit line to the material. If material is not included in the article's Creative Commons license and your intended use is not permitted by statutory regulation or exceeds the permitted use, you will need to obtain permission directly from the copyright holder. To view a copy of this license, visit http://creativecommons. org/licenses/by/4.0/.
\end{abstract}

Open Access This article is licensed under a Creative Commons

(c) The Author(s) 2021 